\title{
THE MAGNETIC ROTATION OF SODIUM VAPOR.
}

By R. W. Wood and H. W. Springsteen.

\section{INTRODUCTION.}

IN I 8 I I Arogo ${ }^{1}$ discovered that rotation of the plane of polarization occurs when plane polarized light is transmitted through quartz in the direction of its optic axis. This was followed by the careful investigations of Biot, ${ }^{2}$ which led him to the deduction of his three well-known laws. That the phenomenon was not confined to solids alone, but occurred also with liquids and gases, was soon made evident by the work of Biot, Sir J. Herschel, Brewster, Faraday and others. Then followed Faraday's discovery ${ }^{3}$ that the plane of polarization is rotated by isotropic substances when placed in a magnetic field.

After the discovery by Fox Talbot, ${ }^{4}$ about 1840 , of the existence of anomalous dispersion, this phenomenon was looked for in the rotatory dispersion of optically-active substances. Biot, ${ }^{5}$ Arndtsen, ${ }^{6}$ Landolt, ${ }^{7}$ Nasini and Gennari, ${ }^{8}$ Cotton ${ }^{9}$ and others observed anomalous rotatory dispersion in various natural active liquids.

It was natural to expect that this anomaly might also exist in magnetically active substances; several investigators have studied various substances with results not altogether concordant. Becquerel ${ }^{10}$ in 1880 observed the effect in oxygen, but others have

1 Arago, Oeuvres Completes, Tom. X., p. 35, I8I 2.

2 Biot, Ann. de Chimie et de Physique, 1815 .

${ }^{3}$ Faraday, Experimental Researches (XIXth series), Vol. III., p. I ; Phil. Trans., p. I, 1846 .

'Talbot, see Tait's Light, 196, and Proc. Roy. Soc. Edinburgh, 1870-71.

${ }^{5}$ Biot, Ann. de Chimie et de Physique (3), 10, p. 5, 1844.

${ }^{6}$ Arndtsen, Ann. de Chimie et de Physique (3), 54, p. $403,1858$.

' Landolt, Beibl., 5, p. 298, r 88 r.

8 Nasini and Gennari, Zeitschr. fur phys. Chemie, I9, p. I13, 1896.

${ }^{9}$ Cotton, Ann. de Chimie et de Physique (7), 8, p. 347, 1876.

${ }_{10}$ Becquerel, Compt. Rend., 90, p. I407, I880. 
been unable to confirm his results. Macaluso and Corbino ${ }^{1}$ worked with sodium vapor and found that the rotation was positive, and abnormally great, on each side of the $D$ absorption bands, but had a small positive value inside the band. Zeeman ${ }^{2}$ also, with a flame between magnet poles, investigated the rotation with highly rarified vapor in the vicinity of the $D$ lines and obtained a positive rotation just outside the two $D$ lines and a very great negative rotation inside each of them, or rather between the components into which the magnetic field divides the line.

Results published by Schmauss in the Annalen du Physik during the year 1903 appeared to show that the rotatory dispersion is anomalous in the vicinity of the absorption band. He worked with alcoholic solutions of the analine dyes, and with didymium glass, obtaining curves not unlike the anomalous dispersion curves of these substances. His work was repeated by Bates ${ }^{3}$ who failed to find any effect whatever due to the addition of the dye, and one of the present writers, has been unable to observe any trace of magnetic rotation in the case of thin plates of solid cyanine in very intense magnetic fields, although a saturated solution of praseodymium chloride appears to show the effect.

In the case of sodium vapor we know positively that the rotation is not anomalous, having the same value for wave-lengths symmetrically situated to the right and left of the $D$ lines.

The subject is of much interest theoretically, for the rotation formula developed from the hypothesis of molecular currents ${ }^{4}$ calls for an anomalous effect, while the one obtained by considering the "Hall-effect," $i$. $c$., the ponderomotive force exerted upon an electron moving in a magnetic field, represents, qualitatively at least, the condition found in the case of sodium vapor.

It was with a view of testing this formula quantitatively that the experiments about to be described were undertaken.

To test a formula we require observations over a fairly wide range of wave-lengths.

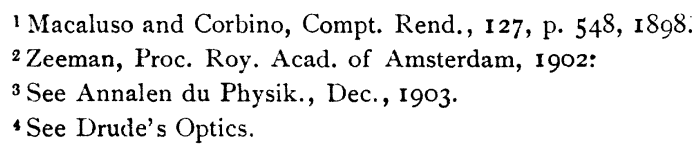


In the course of some work upon the dispersion of the vapor of metallic sodium, it was found by one of us, that the vapor possesses an enormous rotatory power, even for wave-lengths considerably removed from the $D$ lines, when placed in a strong magnetic field.

It was at once recognized that by employing this medium, the rotatory dispersion formula

$$
\grave{\delta}=\frac{\mathrm{I}}{n}\left(\frac{a}{\lambda^{2}}+\frac{b \lambda^{2}}{\left(\lambda^{2}-\lambda_{m}{ }^{2}\right)^{2}}\right)
$$

could be tested.

That it represents the dispersion qualitatively is clear from the occurrence of the term $\left(\lambda^{2}-\lambda_{m}{ }^{2}\right)^{2}$ in the denominator, for $\lambda^{2}-\lambda_{m}{ }^{2}$ changes sign when we cross the absorption band, and it is this change of sign which gives us the anomalous dispersion. In the present case, however, the squaring of the term gives us the same sign on opposite sides of the absorption band, which calls for a dispersion curve of the type shown in Fig. I. A curve of this type was obtained by

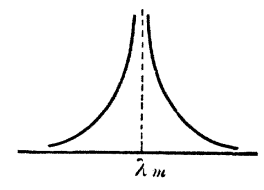

Fig. 1. Cotton, but his observations were made with flames, and the effect could only be traced to a very small distance to the right and left of the $D$ lines.

We can modify the above formula somewhat in the case of sodium vapor as will be shown later on in the paper.

\section{Apparatus and Method.}

The sodium vapor obtained by heating a salt of sodium in a flame is necessarily very rare, and with such a vapor as is well known, the rotation only very near the $D$ lines is perceptible. In order to obtain greater rotations it is obvious that denser vapor must be employed. This was accomplished by heating with a Bunsen flame pure sodium in an exhaused glass tube. This tube, of hard Jena glass, had a length of $25 \mathrm{~cm}$. and an inside diameter of $1.3 \mathrm{~cm}$. Over this and occupying a position midway between the ends of the glass tube was a brass tube $8.2 \mathrm{~cm}$. in length and of such a diameter that it would slide easily over the glass tube. A solid cylin- 
drical piece of pure sodium of the same length as the brass tube, and having a diameter of $0.6 \mathrm{~cm}$. was prepared in the following way. A good-sized piece of sodium was melted in an iron crucible over a Bunsen flame, and the molten metal poured into an iron cylinder. This cylinder had a tight fitting iron piston which, when pressed in by squeezing it in a vice, forced the sodium out through a small hole in the end, and the desired length being then cut off. The sodium stick was then placed in the center of the glass tube as quickly as possible to prevent the moist air from coming in contact with it, and the ends of the tube closed with thin pieces of plate glass, cemented on. The glass ends were tested in the magnetic field employed for the experiment, but no rotation due to them could be detected.

Directly over the sodium and inside the glass tube was a thermos element made of iron and constatin fused together; the terminals passing out through a fine glass tube at one end of the sodium



Fig. 2.

tube. Passing through the other end was a tube of small diameter which was connected to an air-pump and sealed off after exhausting the tube. The vacuum used was about one tenth of a $\mathrm{mm}$. of mercury. The sodium tube was then placed inside a Ruhmhorff magnet as shown in the figure, so that the part of the tube containing the sodium was between the poles of the magnet. The magnet possessed the usual nicol prisms, one at each end, the analyzing one being provided with a rotating arm and scale which could be read to tenths of a degree. The wires from the thermo- 
element passed out alongside the latter nicol, and communicated with a galvanometer and two other thermo-elements, one of iron and copper and the other constantin and copper. These two elements were placed side by side in a kerosene bath and kept at constant temperature. Directly beneath the center of the brass tube was a Bunsen burner, the height of which was so regulated as to produce the required temperature of the vapor, as determined by the scale-reading of the galvanometer. The brass tube served to distribute the heat from the flame more evenly than could otherwise have been done, and by this means the vapor was kept at a very uniform density.

A I Io-volt circuit was connected to the magnet through a double-knife switch, rheostat and ammeter. Since the magnet coils were made of very large wire, a heavy current could be used and the rheostat was adjusted until the current was 35 amperes, and all the observations were made with this current through the magnet. The current, of course, heated the magnet somewhat, but after a short time this heating became fairly steady and with a constant current, the variation in the field-strength introduced no more error than the errors of observation. The strength of the field was determined by measuring the rotation for $D$ light which a tube of carbon bisulphide, $8.2 \mathrm{~cm}$. in length, produced. The average rotation obtained was $16^{\circ} .0$. Assuming 0.042 in minutes of arc as the value of Verdet's constant, this rotation of $16^{\circ}$ corresponds to a field strength of 2,790 C.G.S. units.

Considerable difficulty was experienced in trying to obtain a sufficiently intense beam of light. The light had to pass through various optical parts and also the dense vapor so that finally only a small percentage of the incident light succeeded in getting through the apparatus. As first tried, the light from an arc was brought to a focus just in front of the polarizing nicol, and after passing through this and the sodium vapor, it could be completely extinguished by the analyzing nicol. Just in front of the analyzing nicol was placed a transmission grating of 14,000 lines and when the nicols were parallel, a bright spectrum was produced in the first order. When the nicols were crossed, the spectrum entirely disappeared. The sodium was now slowly heated, and with the 
nicols parallel, the $D$ absorption band slowly appeared, becoming wider and wider as the vapor became denser.

The nicols were now crossed and when the current was turned on, the light was immediately restored for a considerable distance on each side of the $D$ absorption band. It also seemed as if there was a restoration of the light in the part of the spectrum corresponding to the position of the fluted absorption bands, but it was not possible to be sure, with this arrangement of the apparatus. If the analyzing nicol was now slowly turned in a direction which indicated a positive rotation, there appeared two broad black bands, one on each side of the $D$ band. As the nicol was rotated more and more, these bands narrowed up moving closer and closer to the edges of the $D$ band, until finally they reached it. This showed that the rotation was positive on both sides of the $D$ band, and by the readings of the nicol, it was seen that the rotation was very great close to the edges of the $D$ band, but that it rapidly decreased for points further and further away. By using less dense vapor and a reflection grating mounted on a spectrometer, it was possible to see in the second order that the rotation was also positive between the two $D$ lines, but the light here was too faint to permit of a very accurate determination of its amount. It was now necessary to devise some means of determining the wave-length cut out by the black band as the nicol was rotated. To effect this, the light from the arc was focussed on a slit and then made parallel by a collimating lens before it passed through the first nicol. After emerging from the second nicol, it then fell upon a plane reflection grating and into the telescope of the spectrometer. Then the light from a helium tube and a mercury tube was sent through at the same time by means of a small right-angled prism placed on the slit of the collimator, with the intention of using their characteristic lines as reference points from which to measure wave-lengths. But it was found impossible to make the lines appear sufficiently bright and the use of these tubes was abandoned.

Next the sunlight from the heliostat was substituted for the arc, with the hope that the Fraunhofer lines might be sharp enough to be used. But these too, proved to be too indistinct to be employed. 
The next method tried was the following: sunlight was focussed on the slit of a monochromatic illuminator and light of the desired wave-length then transmitted through the first nicol, the sodium and the analyzing nicol in turn. The latter was then rotated until the light appeared to the eye, to be entirely extinguished. By this means the rotation for different wave-lengths was obtained at some distance on either side of the $D$ band, but when settings were made in its vicinity, the source of light could not be made monochromatic enough, even with the slits of the monochromatic illuminator very narrow, since the magnetic rotation in this vicinity is very great, and although the nicol might be turned so as to extinguish light of one wave-length, light of other wave-lengths would still pass through. With this arrangement, however, the rotation was measured for wave-lengths rather distant from the $D$ band and readings were taken until the restored light became too feeble to make the settings accurate.

For the vicinity of the $D$ band another method was found which proved very satisfactory. A beam of sunlight from a heliostat was sent through the nicols and sodium tube and focussed upon the slit of a two-prism spectroscope which possessed a resolving power sufficient to separate the $D$ lines. By this means the Fraunhofer lines were in sharp focus. An auxiliary scale reflected into the field of view, appeared superposed on the solar spectrum.

With the nicols parallel and the vapor of the right density, not only was the $D$ absorption band visible, but also the red and blue channelled absorption bands were very strong. When the nicols were crossed, the field was dark, but when the current was turned on, the spectrum brightened up, not only on each side of the $D$ band, gradually shading off in intensity, but also in the red and blue-green. As many as eight distinct red, and eight blue, bright lines were counted. These bright lines apparently coincided with the red and blue absorption bands of sodium vapor, and showed conclusively that the red and blue absorption bands have also a very marked effect on the magnetic rotation.

The bright blue lines due to magnetic rotation were much fainter than the red and it was noticed that when the vapor was of small density, the bright lines in the red appeared, but no trace was seen 
of those in the blue-green region. This indicates that the rotation is greater for the red lines than for the blue ones. The absorption lines appear, however, in the greenish blue at a smaller vapor density than that necessary for the appearance of the red absorption bands.

The nicol was rotated and the position of the black bands noted with reference to the spectroscope scale. The position of the nicol was read and the temperature of the vapor recorded. The readings were always taken with a constant current through the magnet. Throughout the experiment the temperature of the vapor remained nearly constant. It is clear that the black bands mark the wavelengths, for which the rotation is represented by the angle through which the nicol has been turned from its original position of extinction.

The results obtained are recorded in the following table, which is for wave-lengths on the green side of the absorption band.

\begin{tabular}{c|c|c}
\hline$\lambda$ & & \multicolumn{2}{|c}{$\delta$} \\
\cline { 2 - 3 } & Observed. & \multicolumn{2}{|c}{ Calculated. } \\
\hline 5,886 & $2,371^{\circ}$ & 2,285 \\
5,884 & 1,185 & 1,016 \\
5,876 & 247 & 186 \\
5,875 & 205 & 162 \\
5,870 & 107 & 91 \\
5,861 & 49 & 40.6 \\
5,844 & 14.8 & 15.2 \\
5,835 & 10.8 & 10.9 \\
5,820 & 6.5 & 6.9 \\
5,805 & 4.8 & 4.8 \\
$* 5,770$ & 2 & 2.5 \\
$* 5,725$ & 1.4 & 1.4 \\
$* 5,690$ & 1.2 & 1 \\
$* 5,660$ & 1.0 & - \\
$* 5,630$ & 0.6 & - \\
$* 5,600$ & 0.4 & 0.5
\end{tabular}

The readings marked with an asterisk were made with the monochromatic illuminator, the others by observing the position of the dark band in the continuous spectrum, which appears the moment the second nicol is turned from its position of extinction. The high values for the first five wave-lengths, were obtained by making observations with a grating, using a vapor of small density, and then 
enlarging them to the scale of the density of the vapor used in the other experiments. The rotations on the red side of the $D$ lines are similar for wave-lengths similarly located, in other words the curve is symmetrical. The complete curve is shown in Fig. 3.

It is probable that the complete curve is far from accurate as a whole, for it was constructed from different sets of readings. Moreover the density of the vapor is apt to change during the time occupied in observing and recording a single series.

At the present time experiments are being made by one of us with a photographic method, which it is expected will yield far more accurate results. ${ }^{1}$

Proof of the Rotatory DisPERSION FORMULA.

In the complete formula

$$
\grave{o}=\frac{\mathrm{I}}{n}\left(\frac{a}{\lambda^{2}}+\frac{b \hat{\lambda}^{2}}{\left(\hat{\lambda}^{2}-\lambda_{m}^{2}\right)^{2}}\right)
$$

the first term in the parentheses represents the effect of electrons with periods in the ultra violet. The dispersion of sodium vapor

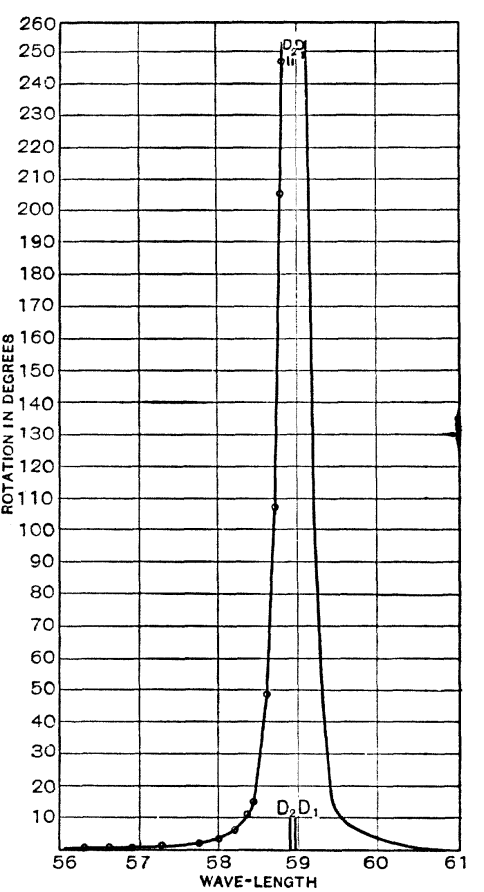

Fig. 3. has been shown by one of the writers to be represented by the formula

$$
n^{2}=\mathrm{I}+\frac{b \lambda^{2}}{\lambda^{2}-\lambda_{m}^{2}},
$$

in other words the electrons which cause the $D$ lines are alone operative. We can thus safely assume that the rotation is influenced only by these same electrons, in other words we may neglect the

1 These measurements have been repeated with a 14 -foot concave grating, not only on each side of, but also between the $D$ lines. The rotating lines in the red and blue green region, to the number of 150 have been accurately measured with the same grating. The results will appear shortly in the Philosophical Magazine.

R. W. W. 
term $a / \lambda^{2}$. The effects contributed by the absorption bands in the blue-green and red can be left out of account as they are but very small. Moreover in the case of sodium vapor $n$ does not differ greatly from unity, except very near the $D$ lines. We can therefore write the formula for the rotation

$$
\grave{\partial}=\frac{b \grave{\lambda}^{2}}{\left(\grave{\lambda}^{2}-\lambda_{m}^{2}\right)^{2}}
$$

and determine the constant $b$ from a single observed rotation.

It is clear that the term $\lambda^{2} /\left(\lambda^{2}-\lambda_{m}{ }^{2}\right)^{2}$ will change with changing wave-length much more rapidly than the term $\lambda^{2} /\left(\lambda^{2}-\lambda_{m}{ }^{2}\right)$ (which occurs in the ordinary dispersion formula); the rotatory dispersion curve will consequently be much steeper than the dispersion curve, as will be readily seen by comparing the curve shown in Fig. 3, with the dispersion curve obtained by Wood for sodium vapor. ${ }^{1}$ In calculating the rotations $\lambda_{m}$ was given the mean value of the $D$ lines 5,893 in the case of the remotor wave-lengths, and the value 5,890 in the case of those near the absorption. This is an approximation method of course, but it was found to work fairly well in the case of the dispersion of the vapor. The calculated results agree fairly well with the observed, except for the immediate vicinity of the $D$ lines, where they are for the most part too low.

A series of readings were taken with improved apparatus, using a diffraction grating, and measuring the position of the band with a filar micrometer. These observations were made as rapidly as possible to avoid changes in the density, which, as has been already said, are probably responsible for most of the errors. The constant was calculated from the rotation observed for wave-length 5,839,

\begin{tabular}{c|c|c|c}
\hline$\lambda$ & \multicolumn{2}{|c|}{$\delta$} & \\
\hline 5,875 & Observed. & Calculated. \\
\hline 5,886 & $95^{\circ}$ & 87.2 & \\
5,875 & 36 & 38.7 & Constant $b=113,382$ \\
5,839 & 23 & 21.7 & $\lambda_{m}=5,893$ \\
5,821 & 9.5 & 9.5 & \\
5,803 & 5 & 5.4 & \\
\hline
\end{tabular}

${ }^{1}$ Phil. Mag., Sept., 1904. 
and the observed and calculated values compared. The results are recorded in the opposite table, and indicate that the rotatory dispersion is probably represented by the formula given above.

More accurate results are expected with the photographic apparatus, which will enable the position of the dark bands to be rapidly recorded for different settings of the nicol. The maesurements can then be made at leisure. 